PROCEEDINGS OF THE

AMERICAN MATHEMATICAL SOCIETY

Volume 141, Number 8, August 2013, Pages 2699-2702

S 0002-9939(2013)11627-8

Article electronically published on April 3, 2013

\title{
LENGTH INEQUALITIES FOR RIEMANN SURFACES
}

\author{
A. F. BEARDON
}

(Communicated by Mario Bonk)

ABStract. We establish inequalities between the lengths of certain closed loops in the triply punctured sphere and in the twice-punctured disc.

\section{INTRODUCTION}

Let $\mathbb{H}$ be the hyperbolic plane $\{x+i y: y>0\}$ with the hyperbolic metric $|d z| / y$ and hyperbolic distance $\rho$. The Uniformisation Theorem states that each hyperbolic Riemann surface $\mathcal{R}$ is conformally equivalent to a quotient space $\mathbb{H} / G$, where $G$ is a discrete group of Möbius transformations (that is, a Fuchsian group) that acts on $\mathbb{H}$. The group $G$ is isomorphic to the fundamental group of $\mathcal{R}$, no element of $G$ (except the identity map) has a fixed point in $\mathbb{H}$, and the hyperbolic metric in $\mathbb{H}$ projects to give a unique metric (the hyperbolic metric) of curvature -1 on $\mathcal{R}$.

Let $\mathcal{R}$ be a hyperbolic Riemann surface which we write as $\mathbb{H} / G$. Let $\zeta$ be a point on $\mathcal{R}$, and let $\alpha$ be a closed loop on $\mathcal{R}$ that starts and ends at $\zeta$. Then $\alpha$ lifts to a curve in $\mathbb{H}$ with endpoints, say, $w$ and $g(w)$, where $g \in G$, and the hyperbolic length $\ell(\alpha)$ of $\alpha$ satisfies $\ell(\alpha) \geqslant \rho(w, g(w))$, with equality when $\alpha$ is the projection of the geodesic segment from $w$ to $g(w)$. Moreover, $\alpha$ is homotopically trivial on $\mathcal{R}$ if and only if $g$ is the identity map in $G$. If $\beta$ is another loop on $\mathcal{R}$, also starting and ending at $\zeta$, then, in a similar way, $\ell(\beta) \geqslant \rho(w, h(w))$ for some $h$ in $G$. The author has proved [2, p. 198] that if $g$ and $h$ generate a non-cyclic group $\langle g, h\rangle$, then, for all $z$ in $\mathbb{H}$,

$$
\sinh \frac{1}{2} \rho(z, g(z)) \sinh \frac{1}{2} \rho(z, h(z)) \geqslant 1 .
$$

This is valid for every hyperbolic $\mathcal{R}$ and every $z$ in $\mathbb{H}$, and it is stronger than what is customarily known as the Collar Lemma. It shows, for example, that providing $\langle g, h\rangle$ is not cyclic,

$$
\sinh \left(\frac{1}{2} \ell(\alpha)\right) \sinh \left(\frac{1}{2} \ell(\beta)\right) \geqslant 1 .
$$

In this paper we establish stronger inequalities of a similar nature when $\mathcal{R}$ is a triply punctured sphere and a twice-punctured disc.

First, we consider $\mathcal{R}$ to be topologically a sphere punctured at the three points $p_{1}, p_{2}$ and $p_{3}$. Now suppose that $\zeta \in \mathcal{R}$ and, for $i=1,2,3$, let $\gamma_{i}$ be a simple closed curve that starts and ends at $\zeta$ and that separates $p_{i}$ from the other two punctures

Received by the editors October 26, 2011.

2010 Mathematics Subject Classification. Primary 30F45; Secondary 30F35, 20H05, 20 H10.

Key words and phrases. Riemann surfaces, hyperbolic metric, punctures. 
$p_{j}$ and $p_{k}$. Now (1), applied to each pair $\gamma_{s}$ and $\gamma_{t}$, yields

$$
\sinh \left(\frac{1}{2} \ell_{1}\right) \sinh \left(\frac{1}{2} \ell_{2}\right) \sinh \left(\frac{1}{2} \ell_{3}\right) \geqslant 1,
$$

where $\ell_{s}$ is the length of $\gamma_{s}$. Our first result strengthens this inequality.

Theorem 1.1. Let $\mathcal{R}$ be a sphere punctured at the three points $p_{1}, p_{2}$ and $p_{3}$. Let $\zeta$ be any point of $\mathcal{R}$ and, for $j=1,2,3$, let $\ell_{j}$ be the length of the shortest closed curve that starts and ends at $\zeta$ and that separates $p_{j}$ from the other two punctures. Then

$$
\sinh \left(\frac{1}{2} \ell_{1}\right) \sinh \left(\frac{1}{2} \ell_{2}\right) \sinh \left(\frac{1}{2} \ell_{3}\right) \geqslant(2 / \sqrt{3})^{3}=1.5396 \cdots .
$$

This bound is attained if and only if $\zeta$ is at the common intersection of the three geodesics $\sigma_{j}$, where $\sigma_{j}$ leaves $p_{j}$ and is orthogonal to the geodesic joining the other two punctures.

Next, we assume that $\mathcal{R}$ is a twice-punctured disc. Here we may assume that $\mathcal{R}=\mathbb{D} \backslash\left\{p_{1}, p_{2}\right\}$, where $\mathbb{D}$ is the open unit disc in $\mathbb{C}$ and $p_{1}$ and $p_{2}$ are in $\mathbb{D}$. Select any point $\zeta$ in $\mathcal{R}$, and let $\ell_{j}, j=1,2$, be the length of the shortest closed curve in $\mathcal{R}$ that starts and ends at $\zeta$, and that separates the puncture $p_{j}$ from the other puncture, and from $\partial \mathbb{D}$. Also, let $\ell$ be the length of the shortest closed geodesic that separates both punctures from $\partial \mathbb{D}$.

Theorem 1.2. For the twice-punctured disc described above,

$$
\sinh \left(\frac{1}{2} \ell_{1}\right) \sinh \left(\frac{1}{2} \ell_{2}\right) \geqslant \cosh ^{2}\left(\frac{1}{4} \ell\right) \cosh \rho(\zeta, \sigma),
$$

where $\sigma$ is the geodesic joining the two punctures.

\section{Some Hyperbolic GeOMETRY}

The conformal isometries of $\mathbb{H}$ are the maps $f(z)=(a z+b) /(c z+d)$, where $a, b, c, d \in \mathbb{R}$ and $a d-b c=1$. Any such $f$ may be represented by the pair of matrices

$$
\pm\left(\begin{array}{ll}
a & b \\
c & d
\end{array}\right)
$$

in $\operatorname{SL}(2, \mathbb{R})$, and $t r^{2}(f)$ is uniquely defined as $(a+d)^{2}$. Next, the displacement function for $f$ is $\rho(z, f(z))$, and this is given by

$$
\sinh \frac{1}{2} \rho(z, f(z))=\frac{|z-f(z)|}{2 \sqrt{\operatorname{Im}[z]} \sqrt{\operatorname{Im}[f(z)]}},
$$

where $z=x+i y$ (see [2, p. 130]).

Now suppose that $f$ has two distinct fixed points on $\mathbb{R} \cup\{\infty\}$. Then $f$ is conjugate to a map $F(z)=k z$, where $k>1$. Then it is easy to see (and well-known) that the displacement function of $F$ attains its minimum value $T_{F}$ on the positive imaginary axis and that $T_{F}$, which is the translation length of $F$, is given by $\operatorname{tr}^{2}(F)=4 \cosh ^{2}\left(\frac{1}{2} T_{F}\right)$. Since all terms here are invariant under conjugation, we see that

$$
\operatorname{tr}^{2}(f)=4 \cosh ^{2}\left(\frac{1}{2} T_{f}\right) .
$$

For more details on these and related facts, see [2]. 


\section{The proof of Theorem 1.1}

As any triply punctured sphere is conformally equivalent to $\mathbb{C}_{\infty} \backslash\{0,1, \infty\}$, we may assume that $\mathcal{R}=\mathbb{C}_{\infty} \backslash\{0,1, \infty\}$. Then (see [1, pp. 277-282]) $\mathcal{R}=\mathbb{H} / \Gamma(2)$, where $\Gamma(2)$, which is a congruence subgroup of the Modular group, is generated by $z \mapsto z+2$ and $z \mapsto z /(2 z+1)$. Further, the hyperbolic quadrilateral in $\mathbb{H}$ with vertices $-1,0,1$ and $\infty$ is a fundamental polygon for $G$. The quotient map $\lambda: \mathbb{H} \rightarrow \mathcal{R}$ is the elliptic modular function, and, using the construction of $\lambda$ given in [1], we may take (with the appropriate interpretation) $p_{1}=0=\lambda(\infty)$, $p_{2}=1=\lambda(0)$ and $p_{3}=\infty=\lambda(1)$. Finally, the stabilizers of $\infty, 0$ and 1 in $\Gamma(2)$ are generated by $g_{1}, g_{2}$ and $g_{3}$, respectively, where

$$
g_{1}(z)=z+2, \quad g_{2}(z)=\frac{z}{2 z+1}, \quad g_{3}(z)=g_{1} g_{2}^{-1}(z)=\frac{3 z-2}{2 z-1} .
$$

Now suppose that $w$ in $\mathbb{H}$ projects to $\zeta$ in $\mathcal{R}$. Then, for $j=1,2,3, \ell_{j}=\sigma\left(w, g_{j}(w)\right)$, so that (2) is equivalent to the assertion that

$$
\inf _{z \in \mathbb{H}}\left(\sinh \frac{1}{2} \rho\left(z, g_{1} z\right) \sinh \frac{1}{2} \rho\left(z, g_{2} z\right) \sinh \frac{1}{2} \rho\left(z, g_{3} z\right)\right)=(2 / \sqrt{3})^{3} .
$$

We shall now establish (6). First, (4) shows that

$$
\sinh \frac{1}{2} \rho\left(z, g_{1}(z)\right) \sinh \frac{1}{2} \rho\left(z, g_{2}(z)\right) \sinh \frac{1}{2} \rho\left(z, g_{3}(z)\right)=\frac{1}{y} \cdot \frac{|z|^{2}}{y} \cdot \frac{|z-1|^{2}}{y}
$$

so that (6) is equivalent to

$$
\inf _{z \in \mathbb{H}} \frac{|z(z-1)|^{2}}{y^{3}}=\left(\frac{2}{\sqrt{3}}\right)^{3} .
$$

If we change the variable to $u+i v=z-\frac{1}{2}$ and write

$$
\Phi(u, v)=\frac{\left(u^{2}+v^{2}-\frac{1}{4}\right)^{2}+v^{2}}{v^{3}},
$$

we see that (6) is equivalent to

$$
\Phi(u, v) \geqslant \Phi\left(0, \frac{1}{2} \sqrt{3}\right)=\left(\frac{2}{\sqrt{3}}\right)^{3} .
$$

We now establish the inequality in (7) (the equality is true). If $v \leqslant \frac{1}{2}$, then $\Phi(u, v) \geqslant v^{2} / v^{3} \geqslant 2>(2 / \sqrt{3})^{3}$. If not, then $v>\frac{1}{2}$ so that

$$
\Phi(u, v) \geqslant \Phi(0, v)=\frac{\left(v^{2}-\frac{1}{4}\right)^{2}+v^{2}}{v^{3}}=\frac{\left(v^{2}+\frac{1}{4}\right)^{2}}{v^{3}}=\varphi(v),
$$

say, with equality if and only if $u=0$. By considering $\varphi^{\prime}(v)$ we see that $\varphi(v)$ attains its minimum when $4 v^{2}=3$, and (6) is proved. This argument shows that equality occurs in (2) if and only if $u=0$ and $v=\sqrt{3} / 2$ or, equivalently, when $z=(1+i \sqrt{3}) / 2$, which is at the intersection of the altitudes of the hyperbolic triangle in $\mathbb{H}$ with vertices 0,1 and $\infty$. 


\section{The proof of Theorem 1.2}

We may assume that $\mathcal{R}=\mathbb{D} \backslash\{a,-a\}$ for some $a$ in $(0,1)$, and we may take $G$ to be generated by

$$
g(z)=z+t, \quad h(z)=\frac{z}{z+1},
$$

where $t>4$. The region $\Sigma$ bounded by the two lines $x= \pm t / 2$ and the two circles $|z \pm 1|=1$ is a fundamental region for $G$ and the sides of $\Sigma$ are paired by $g$ and $h$. An examination of the geometry of the actions of $g$ and $h$ shows that $\ell$ is the translation length of $h^{-1} g$, and if we compute $h^{-1} g$ and use (5) we find that $t=4 \cosh ^{2}(\ell / 4)$.

Now choose any $z$ in $\mathbb{H}$ with $z=x+i y$. Then, from (4) and [2, p. 162],

$$
\begin{aligned}
\sinh \frac{1}{2} \rho(z, g(z)) \sinh \frac{1}{2} \rho(z, h(z)) & =\left(\frac{t}{2 y}\right)\left(\frac{|z|^{2}}{2 y}\right) \\
& =\frac{1}{4} t \cosh \rho(z, \mathcal{I}),
\end{aligned}
$$

where $\mathcal{I}$ is the positive imaginary axis, and this gives (3).

\section{REFERENCES}

[1] Ahlfors, L. V., Complex Analysis, McGraw-Hill (third edition), 1978. MR.510197 (80c:30001)

[2] Beardon, A. F., The Geometry of Discrete Groups, Graduate Texts in Mathematics, No. 91, Springer-Verlag, 1983. MR698777 (85d:22026)

CMS, University of Cambridge, Wilberforce Road, Cambridge CB3 OWB, United KINGDOM 